\title{
Chomsky-Schützenberger-Type Characterization of Multiple Context-Free Languages
}

\author{
Ryo Yoshinaka ${ }^{1 \star}$, Yuichi Kaji ${ }^{2}$, and Hiroyuki Seki ${ }^{2}$ \\ 1 Graduate School of Information Science and Technology, Hokkaido University, \\ ry@ist.hokudai.ac. jp \\ ${ }^{2}$ Graduate School of Information Science, Nara Institute of Science and Technology, \\ $\{$ kaji, seki\}@is.naist.jp
}

\begin{abstract}
It is a well-known theorem by Chomsky and Schützenberger (1963) that every context-free language can be represented as a homomorphic image of the intersection of a Dyck language and a regular language. This paper gives a Chomsky-Schützenberger-type characterization for multiple context-free languages, which are a natural extension of context-free languages, with introducing the notion of multiple Dyck languages, which are also a generalization of Dyck languages.
\end{abstract}

\section{Introduction}

A multiple context-free grammar ( $\mathrm{mcfg})$ is a natural extension of a context-free grammar (cfg). A nonterminal symbol in an mcfg derives tuples of strings by synchronized parallel derivation. The direct derivation relation of an mcfg is defined by a function over tuples of strings (of terminal symbols) such that each component of the function value is defined by a concatenation of some components of arguments and constant strings of terminal symbols with a linearity condition on components of arguments. Let us call such a function linear regular. The language generated by an mcfg is called a multiple context-free language (mcfl).

The generative power of mcfgs is properly larger than cfgs and properly smaller than context-sensitive grammars (csgs). There are several computational models that have the same generative power as mcfgs, e.g., string version of linear context-free rewriting systems, finite copying tree-to-string transducers, string generating context-free hypergraph grammars and local unordered scattered context grammars (see $[2,6]$ for the discussion of these equivalences). Mcfgs share many properties with cfgs such as closure properties. There are other grammatical formalisms of which generative power is between cfgs and csgs such as indexed grammars. In contrast to indexed grammars, the membership problem for an mcfl is solvable in polynomial time in the length of an input string and each mcfl is semilinear. These properties are due to the synchronized parallel derivation realized by linear regular functions. Generally, each component of a

\footnotetext{
* He is now concurrently working in ERATO MINATO Discrete Structure Manipulation System Project, Japan Science and Technology Agency.
} 
tuple of strings appearing in the derivation is not adjacent with one another in the resultant string of terminal symbols. However, these components always share synchronized structure of derivation. To capture this property of mcfls, we will introduce multiple Dyck languages and show a theorem that is an extension of the representation theorem of cfls. It is well-known that any cfl can be represented as a homomorphic image of the intersection of a regular language and a Dyck language (Chomsky-Schützenberger theorem). A Dyck language is the set of well-nested parentheses (brackets). A multiple Dyck language is the set of 'well-nested tuples of parentheses.' The main theorem of this paper is that for a given mcfl $L$, there exists a multiple Dyck language $D$, a regular language $R$ and a homomorphism $h$ such that $L=h(D \cap R)$. As is the same with cfls, this representation theorem for mcfls can be easily lifted to the generator theorem.

The main results of this paper were partially published in and are partially based on Chapter 4 of [3].

\section{Preliminaries}

For an alphabet $\Sigma, \Sigma^{*}$ denotes the set of all strings over $\Sigma$ and $\left(\Sigma^{*}\right)^{m}$ denotes the set of all $m$-tuples of strings over $\Sigma$. The empty string is denoted by $\varepsilon$.

\subsection{Context-Free Grammars}

A context-free grammar (cfg) is a tuple $G=\langle\Sigma, N, P, S\rangle$, where $\Sigma$ is a finite set of terminal symbols, $N$ is a finite set of nonterminal symbols, $P \subseteq N \times(\Sigma \cup N)^{*}$ is a finite set of rules, which are denoted by $A \rightarrow \alpha$ for $A \in N$ and $\alpha \in(\Sigma \cup N)^{*}$, and $S \in N$ is called the start symbol. Elements of $P \cap\left(N \times \Sigma^{*}\right)$ are called terminating rules. $\Rightarrow_{G}$ and $\Rightarrow_{G}^{*}$ denote derivations of one step and any steps (including zero-step), respectively. The language generated by a $\mathrm{cfg} G$, which is called a context-free language (cfl), is the set $L(G)=\left\{w \in \Sigma^{*} \mid S \Rightarrow_{G}^{*} w\right\}$. If $P \subseteq N \times\left(\Sigma^{*} \cup \Sigma^{*} N\right), G$ is called a right-linear grammar and $L(G)$ is called a regular language.

Let $\bar{\Sigma}$ denote an alphabet disjoint from $\Sigma$ that admits a bijection $\overline{(\cdot)}$ from $\Sigma$ to $\bar{\Sigma}$. The Dyck grammar over $\Sigma \cup \bar{\Sigma}$ is the $\operatorname{cfg}$ that has $S$ as its unique nonterminal symbol and whose rules are $S \rightarrow \varepsilon$ and $S \rightarrow \operatorname{SaS} \bar{a}$ for all $a \in \Sigma$. The language generated by a Dyck grammar is called a Dyck language. A string on $\Sigma \cup \bar{\Sigma}$ is well-bracketed if it is an element of the Dyck language. An occurrence of $a \in \Sigma$ and an occurrence of $\bar{a} \in \bar{\Sigma}$ in a well-bracketed string are corresponding if they are derived at the same derivation step. Note that the Dyck grammar is unambiguous. According to the custom, we call elements of $\Sigma \cup \bar{\Sigma}$ parentheses.

Chomsky and Schützenberger [1] gave a characterization of cfls by Dyck languages.

Theorem 1. A language $L$ over $\Sigma$ is context-free if and only if there are an alphabet $\Delta$, a homomorphism $h:(\Delta \cup \bar{\Delta})^{*} \rightarrow \Sigma^{*}$ and a regular language $R$ over $\Delta \cup \bar{\Delta}$ such that $L=h(D \cap R)$ where $D$ is the Dyck language over $\Delta \cup \bar{\Delta}$. 
Theorem 1 can be stated in an even stronger (for the 'only if' direction) form:

Theorem 2. For a given alphabet $\Sigma$, there are an alphabet $\Delta$ and a homomorphism $h:(\Delta \cup \bar{\Delta})^{*} \rightarrow \Sigma^{*}$ such that for any language $L$ over $\Sigma, L$ is context-free if and only if there is a regular language $R$ such that $L=h(D \cap R)$ where $D$ is the Dyck language over $\Delta \cup \bar{\Delta}$.

\subsection{Multiple Context-Free Grammars}

We assume a countably infinite set $X$ of variables. A function from $\left(\Sigma^{*}\right)^{m_{1}} \times \cdots \times$ $\left(\Sigma^{*}\right)^{m_{n}}$ to $\left(\Sigma^{*}\right)^{m}$ is said to be linear regular, if there are $t_{1}, \ldots, t_{m} \in\left(\Sigma \cup\left\{x_{i, j} \in\right.\right.$ $\left.\left.X \mid 1 \leq i \leq n, 1 \leq j \leq m_{i}\right\}\right)^{*}$ such that each variable $x_{i, j}$ occurs at most once in $t_{1} \ldots t_{m}$ and for any $\vec{w}_{i}=\left\langle w_{i, 1}, \ldots, w_{i, m_{i}}\right\rangle \in\left(\Sigma^{*}\right)^{m_{i}}$ with $1 \leq i \leq n$, it holds that

$$
f\left(\vec{w}_{1}, \ldots, \vec{w}_{n}\right)=\left\langle v_{1}, \ldots, v_{m}\right\rangle
$$

where each $v_{k}$ for $k=1, \ldots, m$ is obtained from $t_{k}$ by substituting $w_{i, j}$ for $x_{i, j}$ for all $i$ and $j$. We simply write $f\left(\left\langle x_{1,1}, \ldots, x_{1, m_{1}}\right\rangle, \ldots,\left\langle x_{n, 1}, \ldots, x_{n, m_{n}}\right\rangle\right)=$ $\left\langle t_{1}, \ldots, t_{m}\right\rangle$ to denote the definition of $f$. For example, both $f\left(\left\langle x_{1}, x_{2}\right\rangle\right)=$ $\left\langle a x_{1} b, c x_{2} d\right\rangle$ and $g\left(\left\langle x_{1}, x_{2}\right\rangle,\left\langle y_{1}, y_{2}\right\rangle\right)=\left\langle x_{1} y_{1}, y_{2} x_{2}\right\rangle$ are linear regular functions where $x_{1}, x_{2}, y_{1}, y_{2} \in X$ and $a, b, c, d \in \Sigma$, while $h\left(\left\langle x_{1}\right\rangle\right)=\left\langle x_{1} x_{1}\right\rangle$ is not, since $x_{1}$ appears twice in the right-hand side. $f$ is said to be nonerasing, if every variable in the left-hand side of the definition of $f$ appears in the right-hand side. $f$ is terminal-free, if the right-hand side of its definition contains no symbols from $\Sigma$.

An alphabet $N$ is said to be indexed when we assume a function dim that assigns positive integers to symbols in $N$.

A multiple context-free grammar (mcfg) is a tuple $G=\langle\Sigma, N, F, P, S\rangle$, where

$-\Sigma$ is an (unindexed) alphabet whose elements are called terminal symbols,

$-N$ is an indexed alphabet whose elements are called nonterminal symbols,

- $F$ is a finite set of linear regular functions,

- $P$ is a finite set of rules of the form $A \rightarrow f\left(B_{1}, \ldots, B_{n}\right)$ where $A, B_{1}, \ldots$, $B_{n} \in N$ and $f:\left(\Sigma^{*}\right)^{\operatorname{dim}\left(B_{1}\right)} \times \cdots \times\left(\Sigma^{*}\right)^{\operatorname{dim}\left(B_{n}\right)} \rightarrow\left(\Sigma^{*}\right)^{\operatorname{dim}(A)} \in F$,

$-S \in N$ is called the start symbol whose dimension is 1 .

For a rule $\pi=A \rightarrow f\left(B_{1}, \ldots, B_{n}\right)$, the head and the body of $\pi$ refer to $A$ and $f\left(B_{1}, \ldots, B_{n}\right)$, respectively, and the $\operatorname{rank}$ of $\pi$ is defined to be $\operatorname{rank}(\pi)=n$. If $\operatorname{rank}(\pi)=0$ and $f()=\vec{w}$, we simply write $A \rightarrow \vec{w}$ for $\pi$ with suppressing $f$. If $f$ is terminal-free, $\pi$ is also said to be terminal-free.

For each $A \in N, L_{G}(A)$ is recursively defined as the smallest set of $\operatorname{dim}(A)$ tuples of strings satisfying that if $A \rightarrow f\left(B_{1}, \ldots, B_{n}\right) \in P$ and $\vec{w}_{i} \in L_{G}\left(B_{i}\right)$ for $i=1, \ldots, n$, then $f\left(\vec{w}_{1}, \ldots, \vec{w}_{n}\right) \in L_{G}(A)$. The language $L(G)$ generated by $G$ is the set $\left\{w \in \Sigma^{*} \mid\langle w\rangle \in L_{G}(S)\right\} . L(G)$ is called a multiple context-free language (mcfl). Two grammars $G$ and $G^{\prime}$ are equivalent if $L(G)=L\left(G^{\prime}\right)$.

Example 1. Let $G_{1}$ be the $\operatorname{mcfg}\left\langle\Sigma_{1}, N_{1}, F_{1}, P_{1}, S\right\rangle$ such that $\Sigma_{1}=\{a, b, c, d\}$, $N_{1}=\{S, A, B\}$ with $\operatorname{dim}(S)=1, \operatorname{dim}(A)=\operatorname{dim}(B)=2, F_{1}$ consists of $e$, $f, g$ and the constant functions appearing in the body of rules in $P_{1}$ below 
where $e\left(\left\langle x_{1}, x_{2}\right\rangle,\left\langle y_{1}, y_{2}\right\rangle\right)=\left\langle x_{1} y_{1} x_{2} y_{2}\right\rangle, f\left(\left\langle x_{1}, x_{2}\right\rangle\right)=\left\langle a x_{1}, b x_{2}\right\rangle, g\left(\left\langle x_{1}, x_{2}\right\rangle\right)=$ $\left\langle c x_{1}, d x_{2}\right\rangle$, and $P_{1}=\{S \rightarrow e(A, B), A \rightarrow f(A), A \rightarrow\langle a, b\rangle, B \rightarrow g(B), B \rightarrow$ $\langle c, d\rangle\}$. Let us call the rules in $P_{1} \pi_{1}, \pi_{2}, \ldots, \pi_{5}$ in the order written above. For example, $\langle a, b\rangle \in L_{G_{1}}(A)$ by $\pi_{3},\langle a a, b b\rangle \in L_{G_{1}}(A)$ by $\pi_{2},\langle c, d\rangle \in L_{G_{1}}(B)$ by $\pi_{5}$ and $\langle a a c b b d\rangle \in L_{G_{1}}(S)$ by $\pi_{1}$. We have $L\left(G_{1}\right)=\left\{a^{m} c^{n} b^{m} d^{n} \mid m, n \geq 1\right\}$.

For a nonterminal symbol $A$ of an $\operatorname{mcfg} G$, a series of rule application steps to obtain a tuple of strings of terminal symbols $\vec{w} \in L_{G}(A)$ is called a derivation of $\vec{w}$ in $G$.

By $q$-MCFG $(r)$ we denote the collection of mcfgs $G$ such that $\operatorname{dim}(A) \leq q$ for all $A \in N$ and $\operatorname{rank}(\pi) \leq r$ for all $\pi \in P$. $q-\operatorname{MCFL}(r)$ is the class of mctls generated by grammars in $q$-MCFG $(r)$.

$G$ is said to be nonerasing, if all $f \in F$ are nonerasing. It is known that every $G \in q-\mathrm{MCFG}(r)$ has an equivalent nonerasing grammar in $q$-MCFG $(r)$ [8]. Grammars from 1-MCFG $(r)$ are identified with cfgs.

Proposition 1 (Seki et al. [8] and Rambow and Satta [6]). For $q \geq 1$, $q-\operatorname{MCFL}(r) \subsetneq(q+1)-\operatorname{MCFL}(r)$. For $q \geq 2, r \geq 1, q-\operatorname{MCFL}(r) \subsetneq q-\operatorname{MCFL}(r+1)$ except for 2 -MCFL(2) $=2$-MCFL(3). For $q \geq 1, r \geq 3$ and $1 \leq k \leq r-2$, $q-\operatorname{MCFL}(r) \subseteq(k+1) q-\operatorname{MCFL}(r-k)$.

Proposition 2 (Rambow and Satta [6]). Each family $q$-MCFL $(r)$ for $r \geq 2$ is a substitution closed full AFL. That is, they are closed under homomorphism, inverse homomorphism, intersection with regular languages, union, concatenation, the Kleene plus and substitution.

Proposition 3 (Seki et al. [8]). Let $G \in q-\mathrm{MCFG}(r)$ be given. It is decidable in $O\left(|w|^{q(r+1)}\right)$ time whether $w \in L(G)$ for any $w \in \Sigma^{*}$.

\subsection{Multiple Dyck Languages}

Let $q$ and $r$ be fixed. We define the notion of the multiple Dyck language in $q$-MCFL $(r)$ on an indexed alphabet, where we assume that the maximum dimension of elements of the indexed alphabet does not exceed $r$. For an indexed alphabet $\Delta$, let

$$
\widehat{\Delta}=\left\{a^{[i]}, \bar{a}^{[i]} \mid a \in \Delta, 1 \leq i \leq \operatorname{dim}(a)\right\} .
$$

Definition 1. The multiple Dyck grammar $D_{\Delta}$ on an indexed alphabet $\Delta$ is the mcfg that has nonterminal symbols $S_{m}$ with $\operatorname{dim}\left(S_{m}\right)=m$ for $m \leq q$, among which the start symbol is $S_{1}$, and that has rules of the following three types:

1. all the possible terminal-free rules allowed in $q-\operatorname{MCFG}(r)$;

2. rules of the form $S_{m} \rightarrow f_{a}\left(S_{m}\right)$ where $f_{a}\left(\left\langle x_{1}, \ldots, x_{m}\right\rangle\right)=\left\langle a^{[1]} x_{1} \bar{a}^{[1]}, \ldots\right.$, $\left.a^{[m]} x_{m} \bar{a}^{[m]}\right\rangle$ for $a \in \Delta$ with $\operatorname{dim}(a)=m$;

3. rules of the form $S_{m} \rightarrow f\left(S_{m}\right)$ with $f\left(\left\langle x_{1}, \ldots, x_{m}\right\rangle\right)=\left\langle t_{1}, \ldots, t_{m}\right\rangle$ where each $t_{i}$ is either $x_{i}, x_{i} a^{[1]} \bar{a}^{[1]}$ or $a^{[1]} \bar{a}^{[1]} x_{i}$ for some $a \in \Delta$ of dimension 1 . 
The language $L\left(D_{\Delta}\right)$ is called the multiple Dyck language over $\Delta \cup \bar{\Delta}$.

We note that rules of type 3 are redundant if $r>1$. If $q=1$ and $r>1, L\left(D_{\Delta}\right)$ is indeed the (context-free) Dyck language over $\Delta \cup \bar{\Delta}$.

Every element of tuples in $L_{D_{\Delta}}\left(S_{m}\right)$ is well-bracketed. Moreover pairs of corresponding parentheses in a string from $L\left(D_{\Delta}\right)$ are partitioned into groups each of which consists of exactly $\left\langle a^{[1]}, \bar{a}^{[1]}\right\rangle, \ldots,\left\langle a^{[\operatorname{dim}(a)]}, \bar{a}^{[\operatorname{dim}(a)]}\right\rangle$ for some $a \in \Delta$. If some member of such a group $A$ is inside some member of a group $B$, then all members from $A$ are inside some member of $B$. For example, $b^{[1]} a^{[1]} \bar{a}^{[1]} a^{[2]} \bar{a}^{[2]} b^{[1]}$ $b^{[2]} a^{[3]} \bar{a}^{[3]} \bar{b}^{[2]}$ is allowed, while $b^{[1]} a^{[1]} a^{[1]} \bar{b}^{[1]} a^{[2]} a^{[2]} b^{[2]} a^{[3]} \bar{a}^{[3]} \bar{b}^{[2]}$ is not, where $\operatorname{dim}(a)=3$ and $\operatorname{dim}(b)=2$. The way of combining parentheses is restricted by available means in $q-\mathrm{MCFG}(r)$.

\section{Theorem}

This section discusses Chomsky-Schützenberger type characterization of mcfls.

\subsection{Informal Example of Construction}

We first review an idea of the proof of Theorem 1 by using a simple example. Let $G_{0}$ be the $\operatorname{cfg}\left\langle\Sigma_{0}, N_{0}, P_{0}, S\right\rangle$ where $\Sigma_{0}=\{a, b, c\}, N_{0}=\{S, A, B\}, P_{0}=$ $\{S \rightarrow a A, A \rightarrow b A B, A \rightarrow a, B \rightarrow c\}$. We call the four rules $\pi_{1}, \pi_{2}, \pi_{3}$ and $\pi_{4}$

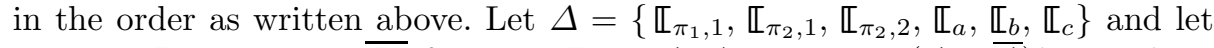
us write $\mathbb{\rrbracket}_{x}$ to denote $\overline{\mathbb{I}_{x}}$ for each $\mathbb{\llbracket}_{x} \in \Delta$. Also let $h:(\Delta \cup \bar{\Delta})^{*} \rightarrow \Sigma_{0}^{*}$ be the homomorphism defined by $h\left(\llbracket_{x}\right)=x$ for $x \in \Sigma_{0}$ and $h(z)=\varepsilon$ for other $z \in \Delta \cup \bar{\Delta}$. Figure 1 shows an example of a derivation tree (called $\left.t_{0}\right)$ in $G_{0}$. Intuitively, $\mathbb{a}_{\pi, i}$ and $\mathbb{1}_{\pi, i}$ mean the left end and the right end of a derivation

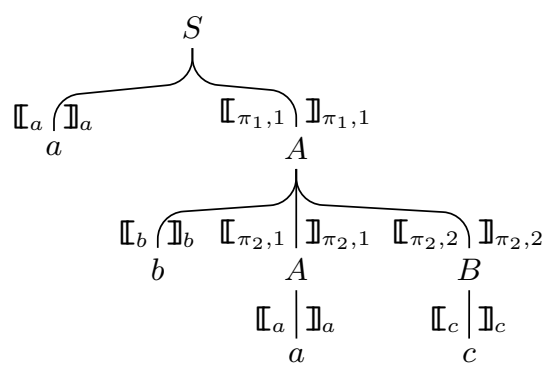

Fig. 1. A derivation tree in $G_{0}$

starting from the $i$-th nonterminal symbol in the body of the rule $\pi$. For $x \in \Sigma_{0}$, a pair $\mathbb{I}_{x}$ and $\mathbb{\rrbracket}_{x}$ denotes $x$. In the figure, paired symbols in $\Delta \cup \bar{\Delta}$ are placed on the left-side and the right-side of each edge. For a tree $t$, let $\alpha(t)$ denote 
the string over $\Delta \cup \bar{\Delta}$ obtained by concatenating these labels in the depth-first left-to-right order. For example,

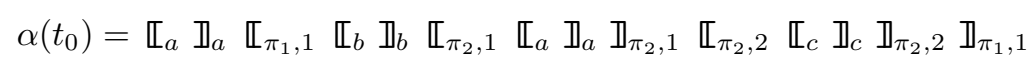

for $t_{0}$ in the figure. For a tree $t$, let yield $(t)$ denote the string obtained by concatenating the labels of leaf nodes of $t$ from left to right. Then, yield $(t)=h(\alpha(t))$ for a derivation tree $t$ in $G_{0}$ and $L\left(G_{0}\right)=h\left(\left\{\alpha(t) \mid t\right.\right.$ is a derivation tree in $\left.\left.G_{0}\right\}\right)$. Therefore, what we should do is to construct a right-linear grammar $G_{R_{0}}$ such that $L\left(G_{R_{0}}\right) \cap D=\left\{\alpha(t) \mid t\right.$ is a derivation tree in $\left.G_{0}\right\}$ in this particular example where $D$ is the Dyck language over $\Delta \cup \bar{\Delta}$. $G_{R_{0}}$ can be defined by considering the finite-state tree traversal that emits $\mathbb{L}_{x}$ and $\mathbb{\rrbracket}_{x}$ when it visits $x \in \Sigma_{0}$, emits $\mathbb{L}_{\pi, i}$ when it visits the $i$-th nonterminal symbol in the body of $\pi$, and emits $\mathbb{1}_{\pi, i}$ when it returns from that nonterminal symbol. Note that nonterminal symbols in $N_{0}$ are used as 'finite states' (nonterminal symbols of $G_{R_{0}}$ ) when the traversal goes down while a new nonterminal symbol $T$ is used when it goes up.

$$
\begin{aligned}
& S \rightarrow \mathbb{I}_{a} \mathbb{1}_{a} \mathbb{I}_{\pi_{1}, 1} A \quad T \rightarrow \mathbb{1}_{\pi_{1}, 1} T \\
& A \rightarrow \mathbb{I}_{b} \mathbb{\rrbracket}_{b} \mathbb{I}_{\pi_{2}, 1} A \quad T \rightarrow \mathbb{1}_{\pi_{2}, 1} \mathbb{L}_{\pi_{2}, 2} B \quad T \rightarrow \mathbb{1}_{\pi_{2}, 2} T \\
& A \rightarrow \mathbb{L}_{a} \mathbb{1}_{a} T \quad B \rightarrow \mathbb{L}_{c} \mathbb{1}_{c} T \\
& T \rightarrow \varepsilon
\end{aligned}
$$

A similar idea can be applied to mcfg. Let $G_{1}$ be the mcfg from Example 1 . Figure 2 shows a tree that illustrates the derivation in the example. (This kind

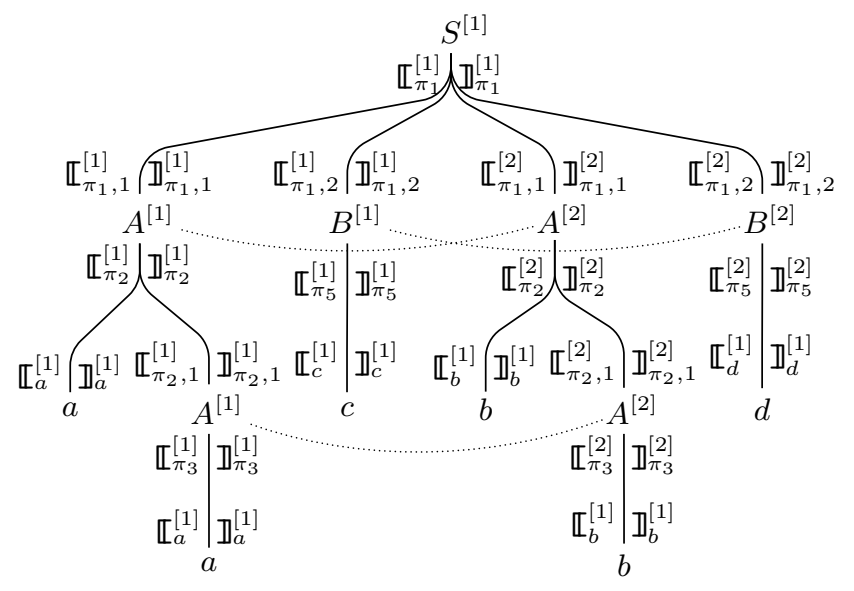

Fig. 2. A derived tree in $G_{1}$

of tree is called a derived tree in mcfg. Here we use derived tree without formal definition since derived tree is not needed in the formal proofs in the rest of this paper.) In the figure, $A^{[j]}(j=1,2)$ denotes a (hypothetical) nonterminal symbol that derives the $j$-th component $w_{j}$ of $\left\langle w_{1}, w_{2}\right\rangle \in L_{G_{1}}(A) . S^{[1]}, B^{[1]}$ and 
$B^{[2]}$ are used in the same purpose. A horizontal arc between $A^{[1]}$ and $A^{[2]}$ means that these two nodes together represent an instance of $A$ in the derivation. Let

$$
\Gamma=\left\{\pi_{1}, \pi_{2}, \ldots, \pi_{5},\left\langle\pi_{1}, 1\right\rangle,\left\langle\pi_{1}, 2\right\rangle,\left\langle\pi_{2}, 1\right\rangle,\left\langle\pi_{4}, 1\right\rangle, a, b, c, d\right\} .
$$

The symbol $\langle\pi, i\rangle(1 \leq i \leq \operatorname{rank}(\pi))$ corresponds to the $i$-th nonterminal symbol in the body of the rule $\pi$. For example, $\left\langle\pi_{1}, 1\right\rangle$ and $\left\langle\pi_{1}, 2\right\rangle$ correspond to $A$ and $B$, respectively. For each $\pi \in P_{1}$, define $\operatorname{dim}(\pi)$ to be the dimension of the head of $\pi$. For each $\pi \in P_{1}$ and $i(1 \leq i \leq \operatorname{rank}(\pi))$, define $\operatorname{dim}(\langle\pi, i\rangle)$ to be the dimension of the $i$-th nonterminal symbol in the body of $\pi$. For each $x \in \Sigma_{1}$, define $\operatorname{dim}(x)=1$. Thus, $\operatorname{dim}\left(\pi_{1}\right)=\operatorname{dim}(a)=\cdots=\operatorname{dim}(d)=1$ and

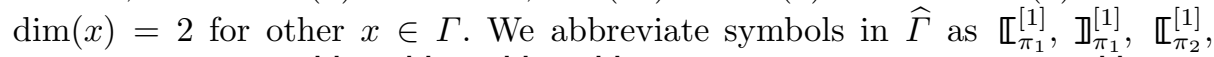

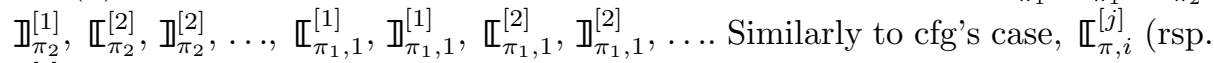
$\mathbb{I}_{\pi, i}^{[j]}$ ) denotes the left (rsp. right) end of a derivation for the $j$-th component of the $i$-th nonterminal symbol in the body of $\pi$. For the $\operatorname{mcfg} G_{1}$, we also have $L\left(G_{1}\right)=h\left(\left\{\alpha(t) \mid t\right.\right.$ is a 'derived tree' in $\left.\left.G_{1}\right\}\right)$ where $h$ is defined similarly to cfg's case. Hence, it suffices to give a right-linear grammar $G_{R_{1}}$ such that $L\left(G_{R_{1}}\right) \cap L\left(D_{\Gamma}\right)=\left\{\alpha(t) \mid t\right.$ is a 'derived tree' in $\left.G_{1}\right\}$. The construction of $G_{R_{1}}$ is a little cumbersome but not difficult:

$$
\begin{aligned}
& S^{[1]} \rightarrow \mathbb{\llbracket}_{\pi_{1}}^{[1]} \mathbb{a}_{\pi_{1}, 1}^{[1]} A^{[1]} \quad T \rightarrow \mathbb{1}_{\pi_{1}, 1}^{[1]} \mathbb{\llbracket}_{\pi_{1}, 2}^{[1]} B^{[1]} \quad T \rightarrow \mathbb{I}_{\pi_{1}, 2}^{[1]} \mathbb{\llbracket}_{\pi_{1}, 1}^{[2]} A^{[2]} \\
& T \rightarrow \mathbb{I}_{\pi_{1}, 1}^{[2]} \mathbb{\llbracket}_{\pi_{1}, 2}^{[2]} B^{[2]} \quad T \rightarrow \mathbb{I}_{\pi_{1}, 2}^{[2]} \mathbb{I}_{\pi_{1}}^{[1]} T \\
& A^{[1]} \rightarrow \mathbb{I}_{\pi_{2}}^{[1]} \mathbb{I}_{a}^{[1]} \mathbb{I}_{a}^{[1]} \mathbb{\llbracket}_{\pi_{2}, 1}^{[1]} A^{[1]} \quad T \rightarrow \mathbb{I}_{\pi_{2}, 1}^{[1]} \mathbb{I}_{\pi_{2}}^{[1]} T \\
& A^{[2]} \rightarrow \mathbb{I}_{\pi_{2}}^{[2]} \mathbb{L}_{b}^{[1]} \mathbb{I}_{b}^{[1]} \mathbb{L}_{\pi_{2}, 1}^{[2]} A^{[2]} \quad T \rightarrow \mathbb{I}_{\pi_{2}, 1}^{[2]} \mathbb{D}_{\pi_{2}}^{[2]} T \\
& A^{[1]} \rightarrow \mathbb{I}_{\pi_{3}}^{[1]} \mathbb{a}_{a}^{[1]} \mathbb{D}_{a}^{[1]} \mathbb{J}_{\pi_{3}}^{[1]} T \quad A^{[2]} \rightarrow \mathbb{\leftarrow}_{\pi_{3}}^{[2]} \mathbb{\leftarrow}_{b}^{[1]} \mathbb{Z}_{b}^{[1]} \mathbb{J}_{\pi_{3}}^{[2]} T \\
& B^{[1]} \rightarrow \mathbb{I}_{\pi_{4}}^{[1]} \mathbb{a}_{c}^{[1]} \mathbb{D}_{c}^{[1]} \mathbb{a}_{\pi_{4}, 1}^{[1]} B^{[1]} \quad T \rightarrow \mathbb{I}_{\pi_{4}, 1}^{[1]} \mathbb{I}_{\pi_{4}}^{[1]} T \\
& B^{[2]} \rightarrow \mathbb{I}_{\pi_{4}}^{[2]} \mathbb{I}_{d}^{[1]} \mathbb{1}_{d}^{[1]} \mathbb{a}_{\pi_{4}, 1}^{[2]} B^{[2]} \quad T \rightarrow \mathbb{1}_{\pi_{4}, 1}^{[2]} \mathbb{I}_{\pi_{4}}^{[2]} T \\
& B^{[1]} \rightarrow \mathbb{I}_{\pi_{5}}^{[1]} \mathbb{I}_{c}^{[1]} \mathbb{I}_{c}^{[1]} \mathbb{I}_{\pi_{5}}^{[1]} T \quad B^{[2]} \rightarrow \mathbb{a}_{\pi_{5}}^{[2]} \mathbb{4}_{d}^{[1]} \mathbb{1}_{d}^{[1]} \mathbb{1}_{\pi_{5}}^{[2]} T \\
& T \rightarrow \varepsilon \text {. }
\end{aligned}
$$

\subsection{Formal Construction}

Let us arbitrarily fix positive integers $q$ and $r$. We now give our ChomskySchützenberger type characterization for $q-\operatorname{MCFL}(r)$. Without loss of generality, we may assume that any $G \in q-\mathrm{MCFG}(r)$ satisfies the following conditions:

- $G$ is nonerasing;

- if $G$ has a rule $A \rightarrow f\left(B_{1}, \ldots, B_{n}\right)$ and $1 \leq i<j \leq n$, then $B_{i} \neq B_{j}$.

Indeed every mcfg in $q-\mathrm{MCFG}(r)$ has an equivalent one in $q-\mathrm{MCFG}(r)$ with this property.

Let $G=\langle\Sigma, N, F, P, S\rangle \in q-\mathrm{MCFG}(r)$ be given. Our goal is to find an indexed alphabet $\Delta$, a right-linear grammar $R$ over $\widehat{\Delta}$, and a homomorphism $h: \widehat{\Delta}^{*} \rightarrow$ $\Sigma^{*}$ such that $L(G)=h\left(L\left(D_{\Delta}\right) \cap L(R)\right)$. 
Let

$$
\Delta=\left\{\mathbb{L}_{a} \mid a \in \Sigma\right\} \cup\left\{\mathbb{L}_{\pi} \mid \pi \in P\right\} \cup\left\{\mathbb{L}_{\pi, i} \mid 1 \leq i \leq \operatorname{rank}(\pi), \pi \in P\right\}
$$

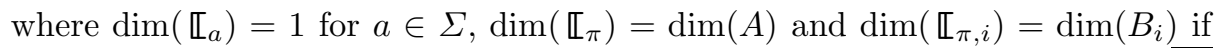
$\pi \in P$ is of the form $A \rightarrow f\left(B_{1}, \ldots, B_{n}\right)$. Hereafter we write $\mathbb{I}_{*}$ instead of $\overline{\mathbb{L}_{*}}$ for each $\overline{\mathbb{I}_{*}} \in \bar{\Delta}$. By $\widetilde{(\cdot)}$ we denote the homomorphism from $\Sigma^{*}$ to $\widehat{\Delta}^{*}$ such that $\widetilde{a}=\mathbb{L}_{a}^{[1]} \mathbb{1}_{a}^{[1]}$.

The nonterminal symbols of the right-linear grammar $R$ is

$$
\{T\} \cup\left\{A^{[k]} \mid A \in N, 1 \leq k \leq \operatorname{dim}(A)\right\}
$$

and the start symbol is $S^{[1]}$. The rules of $R$ are given as follows. Suppose that $G$ has a rule $\pi$ of the form $A \rightarrow f\left(B_{1}, \ldots, B_{n}\right)$ and $f$ is represented as

$$
\begin{aligned}
& f\left(\left\langle x_{1,1}, \ldots, x_{1, m_{1}}\right\rangle, \ldots,\left\langle x_{n, 1}, \ldots, x_{n, m_{n}}\right\rangle\right)=\left\langle t_{1}, \ldots, t_{m}\right\rangle \\
& \text { where } t_{k}=u_{k, 0} x_{i_{k 1}, j_{k 1}} u_{k, 1} \ldots x_{i_{k p_{k}}, j_{k p_{k}}} u_{k, p_{k}} \text { with } u_{k, 0}, \ldots, u_{k, p_{k}} \in \Sigma^{*} \\
& \text { for } k=1, \ldots, m .
\end{aligned}
$$

For each $k=1, \ldots, m$, if $p_{k}=0$, then $R$ has the rule

$$
A^{[k]} \rightarrow \mathbb{\llbracket}_{\pi}^{[k]} \widetilde{u}_{k, 0} \rrbracket_{\pi}^{[k]} T
$$

and otherwise, $R$ has the following $p_{k}+1$ rules:

$$
\begin{aligned}
& A^{[k]} \rightarrow \mathbb{\leftarrow}_{\pi}^{[k]} \widetilde{u}_{k, 0} \mathbb{4}_{\pi, i_{k 1}}^{\left[j_{k 1}\right]} B_{i_{k 1}}^{\left[j_{k 1}\right]}, \\
& T \rightarrow \mathbb{1}_{\pi, i_{k(l-1)}}^{\left[j_{k(l)-1}\right]} \widetilde{u}_{k, l-1} \mathbb{4}_{\pi, i_{k l}}^{\left[j_{k l}\right]} B_{i_{k l}}^{\left[j_{k l}\right]} \text { for } 1<l \leq p_{k}, \\
& T \rightarrow \mathbb{1}_{\pi, i_{k p_{k}}}^{\left[j_{k p_{k}}\right]} \widetilde{u}_{k, p_{k}} \mathbb{D}_{\pi}^{[k]} T \text {. }
\end{aligned}
$$

Moreover $R$ has

$$
T \rightarrow \varepsilon,
$$

which is the unique terminating rule of $R$.

We define the homomorphism $h: \widehat{\Delta}^{*} \rightarrow \Sigma^{*}$ so that for $z \in \widehat{\Delta}$,

$$
h(z)= \begin{cases}a & \text { if } z=\mathbb{\leftarrow}_{a}^{[1]} \text { for some } a \in \Sigma ; \\ \varepsilon & \text { otherwise. }\end{cases}
$$

\subsection{Correctness}

Lemma 1. $L(G) \subseteq h\left(L(R) \cap L\left(D_{\Delta}\right)\right)$.

Proof. By induction we show that if $\left\langle w_{1}, \ldots, w_{m}\right\rangle \in L_{G}(A)$, then there are $v_{1}, \ldots, v_{m} \in \widehat{\Delta}^{*}$ such that $\left\langle v_{1}, \ldots, v_{m}\right\rangle \in L_{D_{\Delta}}\left(S_{m}\right)$ and $A^{[k]} \Rightarrow_{R}^{*} v_{k}$ and $h\left(v_{k}\right)=$ $w_{k}$ for each $k=1, \ldots, m$. 
Suppose that $\left\langle w_{1}, \ldots, w_{m}\right\rangle \in L_{G}(A)$ due to $\pi=A \rightarrow f\left(B_{1}, \ldots, B_{n}\right) \in P$ and $\left\langle w_{i, 1}, \ldots, w_{i, m_{i}}\right\rangle \in L_{G}\left(B_{i}\right)$ for $i=1, \ldots, n$ where $f\left(\left\langle w_{1,1}, \ldots, w_{1, m_{1}}\right\rangle, \ldots\right.$, $\left.\left\langle w_{n, 1}, \ldots, w_{n, m_{n}}\right\rangle\right)=\left\langle w_{1}, \ldots, w_{m}\right\rangle$. Note that the case of $n=0$ provides the basis of the induction.

The induction hypothesis says that for each $i=1, \ldots, n$ we have $v_{i, 1}, \ldots$, $v_{i, m_{i}} \in \widehat{\Delta}^{*}$ such that $\left\langle v_{i, 1}, \ldots, v_{i, m_{i}}\right\rangle \in L_{D_{\Delta}}\left(S_{m_{i}}\right), h\left(v_{i, j}\right)=w_{i, j}$ and $B_{i}^{[j]} \Rightarrow_{R}^{*} v_{i, j}$ for $j=1, \ldots, m_{i}$, where we have $B_{i}^{[j]} \Rightarrow_{R}^{*} v_{i, j} T \Rightarrow_{R} v_{i, j}$ because $T \rightarrow \varepsilon$ is the unique terminating rule of $R$. Let us represent $f$ as

$$
\begin{aligned}
& f\left(\left\langle x_{1,1}, \ldots, x_{1, m_{1}}\right\rangle, \ldots,\left\langle x_{n, 1}, \ldots, x_{n, m_{n}}\right\rangle\right)=\left\langle t_{1}, \ldots, t_{m}\right\rangle \\
& \text { where } t_{k}=u_{k, 0} x_{i_{k 1}, j_{k 1}} u_{k, 1} \ldots x_{i_{k p_{k}}, j_{k p_{k}}} u_{k, p_{k}} \text { with } u_{k, 0}, \ldots, u_{k, p_{k}} \in \Sigma^{*} \\
& \text { for } k=1, \ldots, m .
\end{aligned}
$$

We define $v_{k}$ by

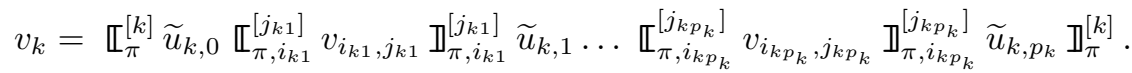

It is easy to see that for each $k, h\left(v_{k}\right)=w_{k}$ and $A^{[k]} \Rightarrow_{R}^{*} v_{k}$ by $B_{i}^{[j]} \Rightarrow_{R}^{*} v_{i, j} T$.

Hence it is enough to show that $\left\langle v_{1}, \ldots, v_{m}\right\rangle \in L_{D_{\Delta}}\left(S_{m}\right)$. Let

$$
\begin{aligned}
v_{i_{k l}, j_{k l}}^{\prime} & \left.=\mathbb{4}_{\pi, i_{k l}}^{\left[j_{k l}\right]} v_{i_{k l}, j_{k l}}\right]_{\pi, i_{k l}}^{\left[j_{k l}\right]} \widetilde{u}_{k, l}, \\
v_{k}^{\prime} & =v_{i_{k 1}, j_{k 1}}^{\prime} \ldots v_{i_{k p_{k}}, j_{k p_{k}}}^{\prime}
\end{aligned}
$$

for $l=1, \ldots, p_{k}$ and $k=1, \ldots, m$. By (1), (2), (3),

$$
v_{k}=\mathbb{\llbracket}_{\pi}^{[k]} \widetilde{u}_{k, 0} v_{k}^{\prime} \mathbb{\rrbracket}_{\pi}^{[k]} .
$$

Applying appropriate rules of type 2 and type 3 of Definition 1 to

$$
\left\langle v_{i, 1}, \ldots, v_{i, m_{i}}\right\rangle \in L_{D_{\Delta}}\left(S_{m_{i}}\right),
$$

for $i=1, \ldots, n$, we have

$$
\left\langle v_{i, 1}^{\prime}, \ldots, v_{i, m_{i}}^{\prime}\right\rangle \in L_{D_{\Delta}}\left(S_{m_{i}}\right)
$$

by (2). Applying to those the rule $S_{m} \rightarrow f^{\prime}\left(S_{m_{1}}, \ldots, S_{m_{n}}\right)$ of type 1 where $f^{\prime}$ is obtained by removing all the occurrences of terminal symbols in the definition of $f$, we get

$$
\left\langle v_{1}^{\prime}, \ldots, v_{m}^{\prime}\right\rangle \in L_{D_{\Delta}}\left(S_{m}\right)
$$

by (3). By (4), appropriate rules of type 3 and type 2 provide

$$
\left\langle v_{1}, \ldots, v_{m}\right\rangle \in L_{D_{\Delta}}\left(S_{m}\right) .
$$

Lemma 2. Suppose that $A^{[k]} \Rightarrow_{R}^{*} w$ and $w$ is well-bracketed. Then there is a rule $\pi \in P$ such that the head of $\pi$ is $A$ and the outermost parentheses of $w$ are just $\mathbb{\llbracket}_{\pi}^{[k]}, \mathbb{I}_{\pi}^{[k]}$. 
Proof. The first rule for deriving $w$ applied to $A^{[k]}$ is either $A^{[k]} \rightarrow \mathbb{I}_{\pi}^{[k]} \widetilde{u}_{k, 0} \mathbb{I}_{\pi}^{[k]} T$ or $A^{[k]} \rightarrow \mathbb{I}_{\pi}^{[k]} \widetilde{u}_{k, 0} \mathbb{\llbracket}_{\pi, i_{k 1}}^{\left[j_{k 1}\right]} B_{i_{k 1}}^{\left[j_{k 1}\right]}$ for some $\pi \in P$. In the former case, only $T \rightarrow \varepsilon$ can be used due to the well-bracketedness of $w$, because all the other rules of $G^{\prime}$ whose heads are $T$ start by a closing parenthesis. In the latter case, the open parenthesis $\mathbb{a}_{\pi}^{[k]}$ must be closed by the succeeding derivation process. The only rule of $G^{\prime}$ for $\mathbb{I}_{\pi}^{[k]}$ is $T \rightarrow \mathbb{I}_{\pi, i_{k p_{k}}}^{\left[j_{k p_{k}}\right]} \widetilde{u}_{k, p_{k}} \mathbb{I}_{\pi}^{[k]} T$. Thus $A^{[k]} \Rightarrow_{G^{\prime}}^{*} \mathbb{I}_{\pi}^{[k]} w^{\prime} \mathbb{I}_{\pi}^{[k]} T \Rightarrow_{G^{\prime}}^{*} w$, where the occurrences of $\mathbb{\llbracket}_{\pi}^{[k]}$ and $\mathbb{I}_{\pi}^{[k]}$ are corresponding. By the same reason for the former case, we must apply the rule $T \rightarrow \varepsilon$ and obtain $w=\mathbb{I}_{\pi}^{[k]} w^{\prime} \mathbb{I}_{\pi}^{[k]}$.

Lemma 3. $h\left(L(R) \cap L\left(D_{\Delta}\right)\right) \subseteq L(G)$.

Proof. We show by induction that whenever $\left\langle w_{1}, \ldots, w_{m}\right\rangle \in L_{D_{\Delta}}\left(S_{m}\right)$ and $A^{[k]} \Rightarrow_{R}^{*} w_{k}$ for $k=1, \ldots, m$ where $m=\operatorname{dim}(A)$, we have $\left\langle h\left(w_{1}\right), \ldots, h\left(w_{m}\right)\right\rangle \in$ $L_{G}(A)$.

Let us consider the derivation of $w_{k}$ in $R$. By Lemma 2, each $w_{k}$ has the form $w_{k}=\mathbb{I}_{\pi_{k}}^{[k]} w_{k}^{\prime} \mathbb{\rrbracket}_{\pi_{k}}^{[k]}$ for some rule $\pi_{k} \in P$ and $w_{k}^{\prime} \in \widehat{\Delta}^{*}$. The outermost parentheses

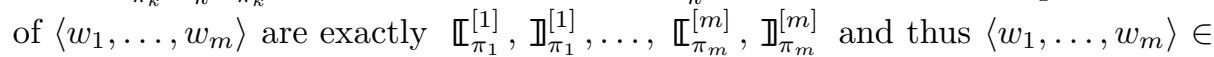
$L_{D_{\Delta}}\left(S_{m}\right)$ implies that $\pi_{1}=\pi_{2}=\cdots=\pi_{m}$. We may hereafter omit the subscript of $\pi_{k}$ as $\pi$. Let $\pi$ be $A \rightarrow f\left(B_{1}, \ldots, B_{n}\right)$ and $f$ represented as

$$
\begin{array}{r}
f\left(\left\langle x_{1,1}, \ldots, x_{1, m_{1}}\right\rangle, \ldots,\left\langle x_{n, 1}, \ldots, x_{n, m_{n}}\right\rangle\right)=\left\langle t_{1}, \ldots, t_{m}\right\rangle \\
\text { where } t_{k}=u_{k, 0} x_{i_{k 1}, j_{k 1}} u_{k, 1} \ldots x_{i_{k p_{k}}, j_{k p_{k}}} u_{k, p_{k}} \text { with } \begin{array}{r}
u_{k, 0}, \ldots, u_{k, p_{k}} \in \Sigma^{*} \\
\text { for } k=1, \ldots, m .
\end{array}
\end{array}
$$

If $p_{k}=0$, the only rule of $R$ that derives $\mathbb{I}_{\pi}^{[k]}$ is $A^{[k]} \rightarrow \mathbb{\llbracket}_{\pi}^{[k]} \widetilde{u}_{k, 0} \mathbb{I}_{\pi}^{[k]} T$ and thus $w_{k}=\mathbb{\llbracket}_{\pi}^{[k]} \widetilde{u}_{k, 0} \mathbb{1}_{\pi}^{[k]}$. If $p_{k} \geq 1$, we have

$$
A^{[k]} \underset{R}{\Rightarrow} \mathbb{4}_{\pi}^{[k]} \widetilde{u}_{k, 0} \mathbb{4}_{\pi, i_{k 1}}^{\left[j_{k 1}\right]} B_{i_{k 1}}^{\left[j_{k 1}\right]} \underset{R}{\stackrel{*}{\Rightarrow}} w_{k} .
$$

Corresponding to the occurrence of $\mathbb{4}_{\pi, i_{k 1}}^{\left[j_{k 1}\right]}, \mathbb{\mathbb { T }}_{\pi, i_{k 1}}^{\left[j_{k 1}\right]}$ must occur in $w_{k}$. The only rule that provides $\mathbb{D}_{\pi, i_{k 1}}^{\left[j_{k 1}\right]}$ is $T \rightarrow \mathbb{1}_{\pi, i_{k 1}}^{\left[j_{k 1}\right]} \widetilde{u}_{k, 1} \mathbb{\leftarrow}_{\pi, i_{k 2}}^{\left[j_{k 2}\right]} B_{i_{k 2}}^{\left[j_{k 2}\right]}$ unless $p_{k}=1$. Thus

$$
\begin{aligned}
& A^{[k]} \underset{R}{\Rightarrow} \mathbb{I}_{\pi}^{[k]} \widetilde{u}_{k, 0} \mathbb{4}_{\pi, i_{k 1}}^{\left[j_{k 1}\right]} B_{i_{k 1}}^{\left[j_{k 1}\right]} \\
& \stackrel{*}{\Rightarrow} \llbracket_{\pi}^{[k]} \widetilde{u}_{k, 0} \mathbb{\llbracket}_{\pi, i_{k 1}}^{\left[j_{k 1}\right]} v_{k, 1} T \\
& \underset{R}{\Rightarrow} \mathbb{\llbracket}_{\pi}^{[k]} \widetilde{u}_{k, 0} \mathbb{\llbracket}_{\pi, i_{k 1}}^{\left[j_{k 1}\right]} v_{k, 1} \mathbb{I}_{\pi, i_{k 1}}^{\left[j_{k 1}\right]} \widetilde{u}_{k, 1} \mathbb{\llbracket}_{\pi, i_{k 2}}^{\left[j_{k 2}\right]} B_{i_{k 2}}^{\left[j_{k 2}\right]} \\
& \stackrel{*}{\Rightarrow} w_{k} \text {. }
\end{aligned}
$$


for some $v_{k, 1}$, which must be well-bracketed. Then we need $\mathbb{D}_{\pi, i_{k 2}}^{\left[j_{k 2}\right]}$ corresponding to the occurrence of $\mathbb{a}_{\pi, i_{k 2}}^{\left[j_{k 2}\right]}$. Repeatedly applying this discussion, we finally get

$$
\begin{aligned}
& A^{[k]} \underset{R}{\Rightarrow} \mathbb{I}_{\pi}^{[k]} \widetilde{u}_{k, 0} \mathbb{L}_{\pi, i_{k 1}}^{\left[j_{k 1}\right]} B_{i_{k 1}}^{\left[j_{k 1}\right]} \\
& \stackrel{*}{\Rightarrow} \mathbb{\llbracket}_{\pi}^{[k]} \widetilde{u}_{k, 0} \mathbb{4}_{\pi, i_{k 1}}^{\left[j_{k 1}\right]} v_{k, 1} T \\
& \stackrel{*}{\Rightarrow} \mathbb{4}_{\pi}^{[k]} \widetilde{u}_{k, 0} \mathbb{4}_{\pi, i_{k 1}}^{\left[j_{k 1}\right]} v_{k, 1} \mathbb{I}_{\pi, i_{k 1}}^{\left[j_{k 1}\right]} \widetilde{u}_{k, 1} \ldots \mathbb{4}_{\pi, i_{k p_{k}}}^{\left[j_{k p_{k}}\right]} v_{i_{k p_{k}}, j_{k p_{k}}} T \\
& \underset{R}{\Rightarrow} \mathbb{I}_{\pi}^{[k]} \widetilde{u}_{k, 0} \mathbb{I}_{\pi, i_{k 1}}^{\left[j_{k 1}\right]} v_{k, 1} \mathbb{I}_{\pi, i_{k 1}}^{\left[j_{k 1}\right]} \widetilde{u}_{k, 1} \ldots \mathbb{4}_{\pi, i_{k p_{k}}}^{\left[j_{k p_{k}}\right]} v_{k, p_{k}} \mathbb{\beth}_{\pi, i_{k p_{k}}}^{\left[j_{k p_{k}}\right]} \widetilde{u}_{k, p_{k}} \mathbb{I}_{\pi}^{[k]} T \\
& \stackrel{*}{\Rightarrow} w_{k} \text {. }
\end{aligned}
$$

This holds for any $p_{k} \geq 1$. By Lemma 2

$$
w_{k}=\mathbb{\llbracket}_{\pi}^{[k]} \widetilde{u}_{k, 0} \mathbb{\llbracket}_{\pi, i_{k 1}}^{\left[j_{k 1}\right]} v_{k, 1} \mathbb{\rrbracket}_{\pi, i_{k 1}}^{\left[j_{k 1}\right]} \widetilde{u}_{k, 1} \ldots \mathbb{\llbracket}_{\pi, i_{k p_{k}}}^{\left[j_{k p_{k}}\right]} v_{k, p_{k}} \mathbb{\rrbracket}_{\pi, i_{k p_{k}}}^{\left[j_{k p_{k}}\right]} \widetilde{u}_{k, p_{k}} \mathbb{\square}_{\pi}^{[k]} .
$$

Let $w_{i, j}=v_{k, l}$ if $x_{i, j}$ occurs as the $l$-th variable in $t_{k}$, i.e., $w_{i_{k l}, j_{k l}}=v_{k, l}$. We note that $B_{i}^{[j]} \Rightarrow_{R}^{*} w_{i, j} T \Rightarrow_{R} w_{i, j}$ and

$$
h\left(w_{k}\right)=u_{k, 0} h\left(w_{i_{k 1}, j_{k 1}}\right) u_{k, 1} \ldots h\left(w_{i_{k p_{k}}, j_{k p_{k}}}\right) u_{k, p_{k}} .
$$

Applying Lemma 2 to each $w_{i, j}$, which must be well-bracketed, we have $w_{i, j}=$ $\mathbb{I}_{\rho_{i, j}}^{[j]} w_{i, j}^{\prime} \rrbracket_{\rho_{i, j}}^{[j]}$ for some rule $\rho_{i, j}$ of $G$. Here the third outermost parentheses of $\left\langle w_{1}, \ldots, w_{m}\right\rangle^{j}$ consist of exactly $\sum_{1 \leq i \leq n} m_{i}$ pairs

$$
\left\langle\mathbb{I}_{\rho_{i, 1}}^{[1]}, \mathbb{I}_{\rho_{i, 1}}^{[1]}\right\rangle, \ldots,\left\langle\mathbb{\leftarrow}_{\rho_{i, m_{i}}}^{\left[m_{i}\right]}, \mathbb{I}_{\rho_{i, m_{i}}}^{\left[m_{i}\right]}\right\rangle \text { for } i=1, \ldots, n .
$$

By $\left\langle w_{1}, \ldots, w_{m}\right\rangle \in L_{D_{\Delta}}\left(S_{m}\right)$, for each $i=1, \ldots, n$ and $j=1, \ldots, m_{i}$, all of

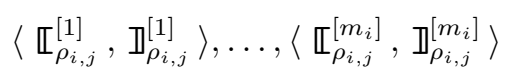

must occur as third outermost parentheses in $\left\langle w_{1}, \ldots, w_{m}\right\rangle$. Recall that the head of the rule $\rho_{i, j}$ is $B_{i}$ and that $B_{i}=B_{i^{\prime}}$ implies $i=i^{\prime}$. Hence $i \neq i^{\prime}$ implies $\rho_{i, j} \neq \rho_{i^{\prime}, j^{\prime}}$ for any $j$ and $j^{\prime}$. Therefore for any $i, j$, it holds that

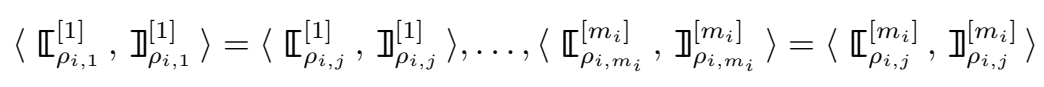

and we have $\rho_{i}$ such that $\rho_{i}=\rho_{i, 1}=\cdots=\rho_{i, m_{i}}$. In the derivation of $\left\langle w_{1}, \ldots, w_{m}\right\rangle \in L_{D_{\Delta}}\left(S_{m}\right)$, at some point the rule $S_{m_{i}} \rightarrow f_{\llbracket_{\rho_{i}}}\left(S_{m_{i}}\right)$ of type 2 , where $f_{\mathbb{I}_{\rho_{i}}}\left(\left\langle x_{1}, \ldots, x_{m}\right\rangle\right)=\left\langle\mathbb{\complement}_{\rho_{i}}^{[1]} x_{1} \mathbb{I}_{\rho_{i}}^{[1]}, \ldots, \mathbb{I}_{\rho_{i}}^{\left[m_{i}\right]} x_{m} \mathbb{I}_{\rho_{i}}^{\left[m_{i}\right]}\right\rangle$, must be applied to $\left\langle w_{i, 1}, \ldots, w_{i, m_{i}}\right\rangle \in L_{D_{\Delta}}\left(S_{m_{i}}\right)$. By the induction hypothesis, we have $\left\langle h\left(w_{i, 1}\right), \ldots\right.$, $\left.h\left(w_{i, m_{i}}\right)\right\rangle \in L_{G}\left(B_{i}\right)$ for $i=1, \ldots, n$. Applying the rule $\pi$ to those tuples, we obtain by (5) and (6)

$$
\begin{aligned}
f\left(\left\langle h\left(w_{1,1}\right), \ldots, h\left(w_{1, m_{1}}\right)\right\rangle, \ldots,\left\langle h\left(w_{n, 1}\right), \ldots, h\left(w_{n, m_{n}}\right)\right\rangle\right) & \\
& =\left\langle h\left(w_{1}\right), \ldots, h\left(w_{m}\right)\right\rangle \in L_{G}(A) .
\end{aligned}
$$


Theorem 3. A language $L$ is in $q-\mathrm{MCFL}(r)$ if and only if there are a multiple Dyck language $D \in q-\operatorname{MCFL}(r)$, a regular language $R$ and a homomorphism $h$ such that

$$
L=h(D \cap R) .
$$

Proof. By Lemmas 1 and 3 and Proposition 2.

\subsection{Generator Theorem}

It is easy to get the stronger Chomsky-Schützenberger-type characterization for $q$-MCFL $(r)$ by the standard technique.

Let

$$
\Delta^{\prime}=\left\{\mathbb{L}_{a} \mid a \in \Sigma\right\} \cup\left\{\mathbb{L}_{m},\left[_{m} \mid 1 \leq m \leq q\right\}\right.
$$

where $\operatorname{dim}(a)=1$ and $\operatorname{dim}\left(\llbracket_{m}\right)=\operatorname{dim}\left(\complement_{m}\right)=m$ and $h^{\prime}:{\widehat{\Delta^{\prime}}}^{*} \rightarrow \Sigma^{*}$ be the homomorphism mapping each $\mathbb{L}_{a}$ to $a$ for $a \in \Sigma$ and other symbols to the empty string.

For a given mcfg $G \in q-\operatorname{MCFG}(r)$, let $\Delta$ and $R$ be the indexed alphabet and the right-linear grammar from Section 3.2, respectively. Let us enumerate

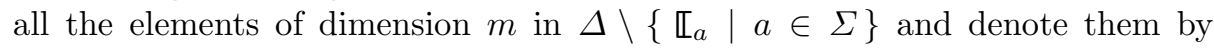
$\mathbb{L}_{m, 1}, \ldots, \mathbb{I}_{m, k_{m}}$ for each $m$. We then define a right-linear grammar $R^{\prime}$ from $R$

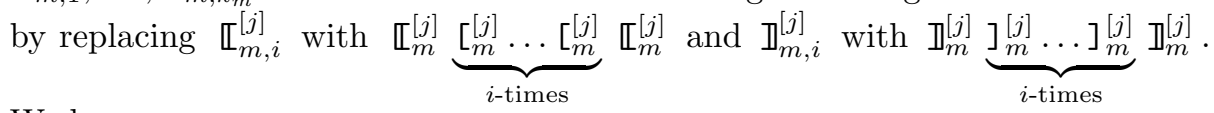

We have

$$
L(G)=h^{\prime}\left(L\left(D_{\Delta^{\prime}}\right) \cap L\left(R^{\prime}\right)\right) .
$$

Corollary 1. There are a multiple Dyck language $D \in q-\mathrm{MCFL}(r)$ and a homomorphism $h$ such that a language $L$ is in $q-\mathrm{MCFL}(r)$ if and only if there is a regular language $R$ such that $L=h(D \cap R)$.

\section{Conclusion}

This paper introduced multiple Dyck languages and then proved a ChomskySchützenberger-type representation theorem for each class $q-\operatorname{MCFL}(r)$ as well as the generator theorem. The literature (e.g. $[4,7])$ has proposed other parameters such as degree and well-nestedness that give further classifications of mcfls. Theorem 3 and Corollary 1 hold for those subclasses as well by accordingly modifying the definition of rules of type 1 of multiple Dyck grammars in Definition 1.

Logical characterizations for several classes of languages have been obtained in the literature. For example, the class of regular languages coincides with the class of languages that are definable in monadic second-order logic (see [9]). Also, the class of cfls is exactly the class of languages definable in an existential second-order logic where the second-order variable ranges only over matching predicates [5]. A matching predicate $M$ is a binary predicate over the set of positions of symbols in a given string such that each position belongs to at most one pair $(i, j)$ satisfying $M(i, j)$ and $M$ is not crossing $((i, j) \in M,(k, l) \in M$ and 
$i<k<j$ imply $i<l<j)$. Intuitively, $M(i, j)$ means that the symbols occurring at the positions $i$ and $j$ form a pair of a left parenthesis and its corresponding right one. This suggests us to extend a matching predicate to a $2 r$-ary predicate $M_{r}$ to express $r$ pairs of left and right parentheses in $\widehat{\Delta}$ of Section 2.3. It is left as future study to give a logic that characterizes mcfls by using these extended matching predicates.

\section{Acknowledgement}

This work was partially supported by the NII joint research project "Open Problems on Multiple Context-Free Grammars" from National Institute of Informatics, Grant-in-Aid for Young Scientists (B-20700124), and a grant from the Global COE Program "Center for Next-Generation Information Technology based on Knowledge Discovery and Knowledge Federation" from the Ministry of Education, Culture, Sports, Science and Technology of Japan.

\section{References}

1. Noam Chomsky and Marcel-Paul Schützenberger. The algebraic theory of contextfree languages. In P. Braffort and D. Hirschberg, editors, Computer Programming and Formal Systems, pages 118-161. North Holland, 1963.

2. Joost Engelfriet. Context-Free Graph Grammars. in Handbook of Formal Languages, volume 3, chapter 3, section 6, Springer, 1997.

3. Yuichi Kaji. Universal recognition problems and a representation theorem using Dyck-type languages for multiple context-free grammars. Bachelor's Thesis, Osaka University, 1991.

4. Makoto Kanazawa. The convergence of well-nested mildly context- sensitive grammar formalisms. An invited talk given at the 14th Conference on Formal Grammar, Bordeaux, France. Slides available at http://research.nii.ac.jp/ kanazawa/.

5. Clemens Lautemann, Thomas Schwentick, and Denis Thérien. Logics for contextfree languages. In Computer Science Logic, Volume 933 of Lecture Notes in Computer Science, pages 205-216. Springer, 1995.

6. Owen Rambow and Giorgio Satta. Independent parallelism in finite copying parallel rewriting systems. Theoretical Computer Science, 223(1-2):87-120, 1999.

7. Hiroyuki Seki and Yuki Kato. On the generative power of multiple context-free grammars and macro grammars. IEICE Transactions, 91-D(2):209-221, 2008.

8. Hiroyuki Seki, Takashi Matsumura, Mamoru Fujii, and Tadao Kasami. On multiple context-free grammars. Theoretical Computer Science, 88(2):191-229, 1991.

9. Wolfgang Thomas. Languages, Automata, and Logic, in Handbook of Formal Languages, volume 3, chapter 7, Springer, 1997.

\section{A Appendix}

The degree of a linear regular function $f:\left(\Sigma^{*}\right)^{m_{1}} \times \cdots \times\left(\Sigma^{*}\right)^{m_{n}} \rightarrow\left(\Sigma^{*}\right)^{m}$ is defined to be $\operatorname{deg}(f)=m+m_{1}+\cdots+m_{n}$ and the degree of an mcfg $G$ is the maximum of $\operatorname{deg}(f)$ for $f \in F$. The functions of rules of type 2 and type 3 
of multiple Dyck grammar $D_{\Sigma}$ in Definition 1 have degree $2 r$ where $r$ is the maximum dimension of letters of $\Sigma$. Indeed we may assume without loss of generality that $\operatorname{deg}(G) \geq 2 \operatorname{dim}(G)$, because of the following lemma.

Lemma 4. If $2 \operatorname{dim}(G)>\operatorname{deg}(G) \geq 2$, there is $G^{\prime}$ equivalent to $G$ such that $\operatorname{dim}\left(G^{\prime}\right)<\operatorname{dim}(G)$ and $\operatorname{deg}\left(G^{\prime}\right) \leq \operatorname{deg}(G)$.

Proof. Without loss of generality, we assume that $G$ is nonerasing. We present a method for eliminating rules whose right hand side has a nonterminal $B$ such that $2 \operatorname{dim}(B)>\operatorname{deg}(G)$. Let

$$
A \rightarrow f\left(B_{1}, \ldots, B_{n}\right) \text { with } f\left(\vec{x}_{1}, \ldots, \vec{x}_{n}\right)=\vec{t}
$$

be a rule such that $2 \operatorname{dim}\left(B_{k}\right)>\operatorname{deg}(G)$ for some $B_{k}$. Since $2\left|\vec{x}_{k}\right|>\operatorname{deg}(G) \geq$ $|\vec{t}|+\left|\vec{x}_{1}\right|+\cdots+\left|\vec{x}_{n}\right|$, there is $t$ in $\vec{t}$ such that $t=t_{1} x_{k, i} u x_{k, j} t_{2}$ for some $x_{k, i}, x_{k, j}$ from $\vec{x}_{k}=\left\langle x_{k, 1}, \ldots, x_{k, m}\right\rangle, u \in \Sigma^{*}$ and $t_{1}, t_{2} \in(\Sigma \cup X)^{*}$. Then we introduce a fresh nonterminal $B_{k}^{\prime}$ with $\operatorname{dim}\left(B_{k}^{\prime}\right)=\operatorname{dim}\left(B_{k}\right)-1$ and replace the rule by

$$
\begin{gathered}
A \rightarrow f^{\prime}\left(B_{1}, \ldots, B_{k-1}, B_{k}^{\prime}, B_{k+1}, \ldots, B_{n}\right) \\
\text { with } f^{\prime}\left(\vec{x}_{1}, \ldots, \vec{x}_{k-1}, \vec{x}_{k}^{\prime}, \vec{x}_{k+1}, \ldots, \vec{x}_{n}\right)=\vec{t}^{\prime}
\end{gathered}
$$

where $\vec{x}_{k}^{\prime}$ is obtained from $\vec{x}_{k}$ by deleting $x_{k, j}$ and $\vec{t}^{\prime}$ is obtained from $\vec{t}$ by replacing $t$ with $t_{1} x_{k, i} t_{2}$. For each rule whose head is $B_{k}$

$$
B_{k} \rightarrow g\left(C_{1}, \ldots, C_{m}\right) \text { with } g\left(\vec{x}_{1}, \ldots, \vec{x}_{m}\right)=\vec{s},
$$

we add the rule

$$
B_{k}^{\prime} \rightarrow g^{\prime}\left(C_{1}, \ldots, C_{m}\right) \text { with } g^{\prime}\left(\vec{x}_{1}, \ldots, \vec{x}_{m}\right)=\vec{s}^{\prime}
$$

where $\vec{s}^{\prime}$ is obtained from $\vec{s}=\left\langle s_{1}, \ldots, s_{m}\right\rangle$ by deleting $s_{j}$ and replacing $s_{i}$ with $s_{i} u s_{j}$. We note that every $C_{l}$ in the body of the new rule satisfies $2 \operatorname{dim}\left(C_{l}\right)<$ $\operatorname{deg}(G)$ by $\operatorname{deg}(G) \geq \operatorname{dim}\left(B_{k}\right)+\operatorname{dim}\left(C_{l}\right)$, and hence the introduced rule will not be a target of repetitive applications of this procedure. It is easy to see that this procedure preserves the language and never increases the degree or the branching factor. 